\title{
SOLUTION OF IRREGULAR SYSTEMS OF PARTIAL DIFFERENTIAL EQUATIONS USING SKELETON DECOMPOSITION OF LINEAR OPERATORS
}

\author{
D.N. Sidorov ${ }^{1,2}$, N.A. Sidorov ${ }^{3}$ \\ ${ }^{1}$ Melentiev Energy Systems Institute SB RAS, Irkutsk, Russian Federation \\ ${ }^{2}$ Irkutsk National Technical University, Irkutsk, Russian Federation \\ ${ }^{3}$ Irkutsk State University, Irkutsk, Russian Federation \\ E-mail: dsidorov@isem.irk.ru, sidorovisu@gmail.com
}

The linear system of partial differential equations is considered. It is assumed that there is an irreversible linear operator in the main part of the system. The operator is assumed to enjoy the skeletal decomposition. The differential operators of such system are assumed to have sufficiently smooth coefficients. In the concrete situations the domains of such differential operators are linear manifolds of smooth enough functions with values in Banach space. Such functions are assumed to satisfy additional boundary conditions. The concept of a skeleton chain of linear operator is introduced. It is assumed that the operator generates a skeleton chain of the finite length. In this case, the problem of solution of a given system is reduced to a regular split system of equations. The system is resolved with respect to the highest differential expressions taking into account certain initial and boundary conditions. The proposed approach can be generalized and applied to the boundary value problems in the nonlinear case. Presented results develop the theory of degenerate differential equations summarized in the monographs MR 87a:58036, Zbl 1027.47001.

Keywords: ill-posed problems; Cauchy problems; irreversible operator; skeleton decomposition; skeleton chain; boundary value problems.

\section{Introduction}

Consider linear equation

$$
\mathbf{B} \mathbf{L}\left(\frac{\partial}{\partial x}\right) u=\mathbf{L}_{1}\left(\frac{\partial}{\partial x}\right) u+f(x)
$$

where linear bounded operator $\mathbf{B}$ acting from linear space $E$ to $E$ has no inverse operator. Differential operators

$$
\begin{aligned}
\mathbf{L}\left(\frac{\partial}{\partial x}\right) & =\frac{\partial^{n}}{\partial t^{n}}+\sum_{k_{0}+k_{1}+\cdots+k_{m} \leq n-1} a_{k_{0} \ldots k_{m}}(x) \frac{\partial^{k_{0}+\cdots+k_{m}}}{\partial t^{k_{0}} \partial x_{1}^{k_{1}} \ldots x_{m}^{k_{m}}}, \\
\mathbf{L}_{1}\left(\frac{\partial}{\partial x}\right) & =\sum_{k_{0}+k_{1}+\cdots+k_{m} \leq n_{1}} b_{k_{0} \ldots k_{m}}(x) \frac{\partial^{k_{0}+\cdots+k_{m}}}{\partial t^{k_{0}} \partial x_{1}^{k_{1}} \ldots x_{m}^{k_{m}}}, \quad n_{1}<n .
\end{aligned}
$$

Here coefficients $a_{k_{0} \ldots k_{m}}: \Omega \subset \mathbb{R}^{m+1} \rightarrow \mathbb{R}^{1}, b_{k_{0} \ldots k_{m}}: \Omega \subset \mathbb{R}^{m+1} \rightarrow \mathbb{R}^{1}$ are sufficiently smooth and defined in $\bar{\Omega}, 0 \in \Omega$. The domains of definition of operators $\mathbf{L}, \mathbf{L}_{1}$ consist of linear manifolds $E_{\partial}$ of sufficiently smooth functions in $\Omega$ with their values in $E$, which satisfy certain system of homogeneous boundary conditions. An abstract function $f: \Omega \subset$ $\mathbb{R}^{m+1} \rightarrow E$ of argument $x=\left(t, x_{1}, \ldots, x_{m}\right)$ is assumed to be given and the problem is to find the solution $u: \Omega \subset \mathbb{R}^{m+1} \rightarrow E_{\partial}$. 
The operator $\mathbf{B}$ is assumed to be independent of $x$. If the operator $\mathbf{B}$ has an inverse bounded operator then equation (1) is called regular and otherwise it is called irregular equation. If $E=\mathbb{R}^{N}$ and $\operatorname{det} B \neq 0$, then (1) is the system of linear partial differential equations (PDE) of Kovalevskaya type, and we have a well known regular problem of the PDE theory. The foundation of many branches of modern general theory of PDE systems was constructed by I.G. Petrovskii [1]. In regular case the initial conditions for (1) can be defined as follows

$$
\left.\frac{\partial^{i} u}{\partial t^{i}}\right|_{t=0}=\varphi_{i}\left(x_{1}, \ldots, x_{m}\right), i=0,1, \ldots, n-1 .
$$

Here functions $\varphi_{i}$ are analytical functions in $\Omega$. If $f$ is an analytic function of $t, x_{1}, \ldots, x_{m}$ in $\Omega$, then the Cauchy problem (1), (2) is not only solvable but also well-posed in class of analytic functions.

The well-posedness of the Cauchy problem is a challenging issue even for linear PDE systems in spaces of non-analytic functions. They are usually solved in a class of functions satisfying certain estimates [1].

Irregular models enable study of systems behavior in critical situations [2]. At present, the basis of relevant theory is constructed for certain classes of equations. For example, the theory and numerical methods for differential-algebraic equations has been constructed.

The intensive studies of more complex theory of irregular PDE and abstract irregular differential operator equations are conducted but there are still a lot of unexplored problems.

If $\mathbf{B}$ is a normally solvable operator, $x \in \mathbb{R}^{1}$ then the approach in the theory of equations of the form (1) can be based on the splitting of the Banach space into a direct sum in accordance to Jordan structure of the operator B [3-5] and G.A. Sviridyuk's results from the theory of semigroups with kernels [6]. These approaches are applied to various problems $[7,8]$.

In this field analytical methods were proposed for constructing classical and generalized solutions of the Cauchy problem for ordinary operator-differential equations with $x \in \mathbb{R}^{1}$ in Banach spaces with irreversible operator in the main part.

The theory of irregular operator-differential PDEs in Banach spaces in the multidimensional case with $x \in \mathbb{R}^{n}, n \geq 2$, is to be constructed. There are only initial results in this field published in preprints. Therefore, the construction of the general theory of equations of the form (1) with irreversible operator $\mathbf{B}$ is of theoretical interest. It is also important for the state of the art mathematical models of compex systems $[2,9,10]$.

It is to be outlined that classical initial Cauchy conditions (2) for equation (1) play very limited role. Indeed, because of the irreversibility the operator $\mathbf{B}$ time direction is characteristic and functions $\varphi_{i}$ can not be arbitrary selected in the initial conditions (2)! Then there appears a question of reasonable formulation and methods of solution of non-classic boundary problems, taking into account the structure of the operator $\mathbf{B}$. The objective of the present work is to solve this problem. In Sec. 2 and Sec. 3 this problem is solved for irreversible operator $\mathbf{B}$ which enjoy skeleton decomposition $\mathbf{B}=\mathbf{A}_{1} \mathbf{A}_{2}$, where $\mathbf{A}_{1} \in \mathcal{L}\left(E \rightarrow E_{1}\right), \mathbf{A}_{2} \in \mathcal{L}\left(E_{1} \rightarrow E\right)$, and $E_{1}$ is a normed space.

The remainder of the paper is organized as follows. Sec. 1 presents an introduction concerning the skeleton chains of linear operators using results [4]. The concept of regular and singular skeleton chains is introduced. It is proved that the operator $\mathbf{B}$ must be nilpotent in case of singular skeleton chain. In Sec. 2 it is assumed that the noninvertible 
operator $\mathbf{B}$ generates a skeleton chain of linear operators of finite length $p$ and it is demonstrated that irregular equation (1) can be reduced to the recurrent sequence of $p+1$ equations. It is to be noted that each equation of this sequence is regular under the natural restrictions on differential operators $\mathbf{L}, \mathbf{L}_{1}$ and certain initial-boundary conditions. Therefore if the operator $\mathbf{B}$ has a skeleton chain of length $p$ then the solution of irregular equation (1) can be reduced to a regular system from the $(p+1)$-th equation.

The proposed approach can be employed for wide range of concrete problems (1) due to finite length of the skeleton chain of the finite-dimensional operator $\mathbf{B}$.

The formulas connecting the solution of (1) with the solution of reduced regular system are derived. This result allows us in Sec. 1 to set new well-posed non-classic boundary conditions for (1) for which the equation enjoys a unique solution what is demonstrated in Sec. 2. For applications, it is important that this solution can be found by solving the sequence of regular problems proposed in this paper. Corresponding results and examples are given in Sec. 3 and Sec. 4.

\section{Skeleton Chains of Linear Operator}

Let $\mathbf{B} \in \mathcal{L}(E \rightarrow E)$, and $\mathbf{B}=\mathbf{A}_{1} \mathbf{A}_{2}$, where $\mathbf{A}_{2} \in \mathcal{L}\left(E \rightarrow E_{1}\right), \mathbf{A}_{1} \in \mathcal{L}\left(E_{1} \rightarrow E\right)$, and $E_{1}, E$ are linear normed spaces. The following definitions can be introduced.

A decomposition $\mathbf{B}=\mathbf{A}_{1} \mathbf{A}_{2}$ is called a skeleton decomposition of the operator $\mathbf{B}$. Introduce a linear operator $\mathbf{B}_{1}=\mathbf{A}_{2} \mathbf{A}_{1}$. Obviously $\mathbf{B}_{1} \in \mathcal{L}\left(E_{1} \rightarrow E_{1}\right)$. If the operator $\mathbf{B}_{1}$ has a bounded inverse or it is null operator acting from $E_{1}$ to $E_{1}$, then $\mathbf{B}$ generates $a$ skeleton chain $\left\{\mathbf{B}_{1}\right\}$ of length 1 . Then the operator $\mathbf{B}_{1}$ can be called a skeleton-attached operator to the operator $\mathbf{B}$. This chain is called singular if $\mathbf{B}_{1}=0$ and regular if $\mathbf{B}_{1} \neq 0$. If $\mathbf{B}_{1}$ is an irreversible non-null operator then it is assumed to have a skeleton decomosition $\mathbf{B}_{1}=\mathbf{A}_{3} \mathbf{A}_{4}$, where $\mathbf{A}_{4} \in \mathcal{L}\left(E_{1} \rightarrow E_{2}\right), \mathbf{A}_{3} \in \mathcal{L}\left(E_{2} \rightarrow E_{1}\right)$, and $E_{2}$ is new linear normed space. Obviously in this case $\mathbf{A}_{2} \mathbf{A}_{1}=\mathbf{A}_{3} \mathbf{A}_{4}$ and an operator $\mathbf{B}_{2}=\mathbf{A}_{4} \mathbf{A}_{3} \in \mathcal{L}\left(E_{2} \rightarrow E_{2}\right)$ can be introduced. If it turns out that $\mathbf{B}_{2}$ has a bounded inverse or $\mathbf{B}_{2} \equiv 0$, then $\mathbf{B}$ has a skeleton chain $\left\{\mathbf{B}_{1}, \mathbf{B}_{2}\right\}$ of length 2. The chain $\left\{\mathbf{B}_{1}, \mathbf{B}_{2}\right\}$ is singular if $\mathbf{B}_{2}=0$ and regular otherwise. Also, the third case is possible: operator $\mathbf{B}_{2}$ is irreversible non null operator. Then chain length is greater than 2 and one should continue chain's construction.

Indeed, this process can be continued for a number of linear operators by introduction of the normed linear spaces $E_{i}, i=1, \ldots, p$ and by bounded operators construction $\mathbf{A}_{2 i} \in$ $\mathcal{L}\left(E_{i-1} \rightarrow E_{i}\right), \mathbf{A}_{2 i-1} \in \mathcal{L}\left(E_{i} \rightarrow E_{i-1}\right)$, which satisfy the following equalities

$$
\mathbf{A}_{2 i} \mathbf{A}_{2 i-1}=\mathbf{A}_{2 i+1} \mathbf{A}_{2 i+2}, i=1,2, \ldots, p-1 .
$$

Equation (3) defines a sequence of linear operators $\left\{\mathbf{B}_{1}, \ldots, \mathbf{B}_{p}\right\}$ as follows

$$
\mathbf{B}_{i}=\mathbf{A}_{2 i} \mathbf{A}_{2 i-1}, i=1,2, \ldots, p .
$$

Obviously $\mathbf{B}_{i} \in \mathcal{L}\left(E_{i} \rightarrow E_{i}\right)$. Here the operator $\mathbf{B}_{p}$ either has a bounded inverse or $\mathbf{B}_{p}$ is a null operator acting from $E_{p}$ to $E_{p}$. This process can be formalized as the following definition.

Definition 1. Let $\mathbf{B}=\mathbf{A}_{1} \mathbf{A}_{2}$ and operators $\left\{\mathbf{A}_{i}\right\}_{i=1}^{2 p}$ satisfy (3). Let operators $\left\{\mathbf{B}_{1}, \ldots, \mathbf{B}_{p}\right\}$ be defined by $(4)$, operators $\left\{\mathbf{B}_{1}, \ldots, \mathbf{B}_{p-1}\right\}$ be noninvertible, and operator $\mathbf{B}_{p}$ have a bounded inverse or be a null operator acting from $E_{p}$ to $E_{p}$. Then the operator 
$\mathbf{B}$ generates a skeleton chain of linear operators $\left\{\mathbf{B}_{1}, \ldots, \mathbf{B}_{p}\right\}$ of length $p$. If $\mathbf{B}_{p} \neq 0$ then the chain is regular, if $\mathbf{B}_{p}=0$ then the chain is called singular. Operators $\left\{\mathbf{B}_{1}, \ldots, \mathbf{B}_{p}\right\}$ are called skeleton-attached to operator $\mathbf{B}$.

The most important linear operators generating skeleton chains of the finite lenghts are given below:

1. Let $E=\mathbb{R}^{m}$, then a square matrix $\mathbf{B}: \mathbb{R}^{m} \rightarrow \mathbb{R}^{m}$ with $\operatorname{det} \mathbf{B}=0$ obviously has skeleton chain $\left\{\mathbf{B}_{1}, \ldots, \mathbf{B}_{p}\right\}$ of decreasing dimentions. The final matrix $\mathbf{B}_{p}$ will be regular or null matrix, $\operatorname{det} \mathbf{B}_{i}=0, i=1, \ldots, p-1$.

2. Let $E$ be an infinite dimentional normed space, then a finite operator $\mathbf{B}=\sum_{i=1}^{n}\left\langle\cdot, \gamma_{i}\right\rangle z_{i}$, where $\left\{z_{i}\right\} \in E, \gamma_{i} \in E^{*}$ has a skeleton chain consisting of finite number of matrices $\left\{\mathbf{B}_{1}, \ldots, \mathbf{B}_{p}\right\}$ of decreasing dimentions. Here $\mathbf{B}_{1}=\left\|\left\langle z_{i}, \gamma_{j}\right\rangle\right\|_{i, j=1}^{n}$ is the first element of this chain, $\operatorname{det} \mathbf{B}_{i}=0, i=1, \ldots, p-1$. $\mathbf{B}_{p}$ is a null matrix or $\operatorname{det} B_{p} \neq 0$.

Here according to Definition 1 the length of the chain $p=1$ if $\operatorname{det}\left[\left\langle z_{i}, \gamma_{j}\right\rangle\right]_{i, j=1}^{n} \neq 0$ or $\left\langle z_{i}, \gamma_{j}\right\rangle=0, i, j=1,2, \ldots, n$. In general case the chain always consists of finite number of matrices.

Using (3), (4) and Definition 1 the following result can be formulated.

Lemma 1. If the operator $\mathbf{B}$ has a skeleton chain of length $p$ then

$$
\mathbf{B}^{n}=\mathbf{A}_{1} \mathbf{A}_{3} \ldots \mathbf{A}_{2 n-1} \mathbf{B}_{n-1} \mathbf{A}_{2 n-2} \mathbf{A}_{2 n-4} \ldots \mathbf{A}_{2}, n=1, \ldots, p+1,
$$

where $\mathbf{B}_{1}, \mathbf{B}_{2}, \ldots \mathbf{B}_{p}$ are elements of the skeleton chain of the operator $\mathbf{B}$.

From Lemma 1 it follows

Corollary 1. If the operator $\mathbf{B}$ has a singular skeleton chain of length $p$, then $\mathbf{B}$ is a nilpotent operator of index $p+1$.

To proof the Corollary it is sufficient to put $n=p+1$ and demonstrate that $\mathbf{B}^{p+1}$ is a null operator because $\mathbf{B}_{p}$ is a null operator due to the above introduced definition of a singular skeleton chain.

\section{Reduction of Abstract Irregular Equation to the Sequence of Regular Equations}

The operator $\mathbf{B}$ and linear operators $\left\{\mathbf{A}_{i}\right\}_{i=1}^{2 p}$ from a skeleton chain of the operator $\mathbf{B}$ are assumed to be independent of $x$ and commutative with linear operators $\mathbf{L}$ and $\mathbf{L}_{1}$. In this paragraph for sake of clarity it is assumed that operators $\mathbf{L}$ and $\mathbf{L}_{1}$ can be different from the introduced above differential operators $\mathbf{L}\left(\frac{\partial}{\partial x}\right), \mathbf{L}_{1}\left(\frac{\partial}{\partial x}\right)$ and equation can be considered in abstract form

$$
\mathbf{B L} u=\mathbf{L}_{1} u+f .
$$

Equation (1) can be considered as a special case of equation (6). Obviously, the introduced commutativity condition is fulfilled for (1) with linear operator $\mathbf{B}$ independent of $x$ and introduced differential operators $\mathbf{L}\left(\frac{\partial}{\partial x}\right), \mathbf{L}_{1}\left(\frac{\partial}{\partial x}\right)$. 
Let us reduce equation (6) to a system of $p+1$ equations which are regular in certain conditions imposed on operators $\mathbf{L}, \mathbf{L}_{1}$. Let us start with the simple case when $p=1$. Introduce a system of two equations

$$
\begin{gathered}
\mathbf{B}_{1} \mathbf{L} u_{1}=\mathbf{L}_{1} u_{1}+\mathbf{A}_{2} f, \\
\mathbf{L}_{1} u=-f+\mathbf{A}_{1} \mathbf{L} u_{1} .
\end{gathered}
$$

where $u \in E$ and $u_{1} \in E_{1}$. The decomposed system $(7)-(8)$ can be obtained by formal multiplication of (6) by the operator $\mathbf{A}_{2}$ from the skeleton decomposition of the operator $\mathbf{B}$ and making notation $u_{1}=\mathbf{A}_{2} u$.

It is to be noted that system (7), (8) is splitted and $\mathbf{B}_{1}$ is an invertible operator. Therefore if the operators $\mathbf{B}_{1} \mathbf{L}-\mathbf{L}_{1}$, and $\mathbf{L}_{1}$ have bounded inverse operators then a unique solution can be constructed. Of course without additional conditions there remains a question: Does a constructed solution $u(x)$ satisfy $(6)$ ?

Let us introduce two lemmas establishing the link between (6) and system (7), (8) to answer that question.

Lemma 2. Let $u^{*}$ satisfy (6) and operator $\mathbf{L}_{1}$ have left inverse. Then a pair $u_{1}^{*}=A_{2} u^{*}, u^{*}$ satisfies (7), (8).

Proof. Based on conditions of the Lemma the following equality is satisfied

$$
\mathbf{A}_{1} \mathbf{A}_{2} \mathbf{L} u^{*}=\mathbf{L}_{1} u^{*}+f
$$

From (9) because of commutativity condition the following equality is valid

$$
\mathbf{A}_{1} \mathbf{L} \mathbf{A}_{2} u^{*}=\mathbf{L}_{1} u^{*}+f
$$

and

$$
\mathbf{A}_{2} \mathbf{A}_{1} \mathbf{L} \mathbf{A}_{2} u^{*}=\mathbf{L}_{1} \mathbf{A}_{2} u^{*}+\mathbf{A}_{2} f .
$$

The latter equality demonstrates that $u_{1}^{*}=\mathbf{A}_{2} u^{*}$ is a solution of (7). Substitution $u_{1}^{*}$ into the right hand side of (8) yields the following equation with respect to $u$ with the known right hand side

$$
\mathbf{L}_{1} u=-f+\mathbf{A}_{1} \mathbf{A}_{2} \mathbf{L} u^{*} .
$$

Here the operators commutativity property is employed. The solution exists for such equation. Indeed, due to (9) right hand side of the equation is equal to $\mathbf{L}_{1} u^{*}$. Hence for given $u^{*}$ and $u_{1}^{*}=\mathbf{A}_{2} u^{*}$ the right hand side belongs to the range of the operator $\mathbf{L}_{1}$. Therefore, $\mathbf{L}_{1} u=\mathbf{L}_{1} u^{*}$. Since the operator $\mathbf{L}_{1}$ has left inverse, then $u^{*}$ is a unique solution to (8) for $u_{1}=\mathbf{A}_{2} u^{*}$. Lemma 2 is proved.

Lemma 3. Suppose that a pair $\left(u_{1}^{*}, u^{*}\right)$ is a solution to (7), (8). Let the operator $\mathbf{L}_{1}$ have right inverse $\mathbf{L}_{1}^{-1}$. Then element $u^{*}$ satisfies (6).

Proof. The element $-f+\mathbf{A}_{1} \mathbf{L} u_{1}^{*}$ belongs to the range of operator $\mathbf{L}_{1}$, because a pair $u_{1}^{*}, u^{*}$ satisfies (7), (8). Hence $u^{*}=\mathbf{L}_{1}^{-1}\left(-f+\mathbf{A}_{1} \mathbf{L} u_{1}^{*}\right)$ because $-f+\mathbf{A}_{1} \mathbf{L} u_{1}^{*} \in \mathcal{R}\left(\mathbf{L}_{1}\right)$, where $\mathcal{R}\left(\mathbf{L}_{1}\right)$ is the range of the operator $\mathbf{L}_{1}$. It is to be demonstrated that the constructed 
element $u^{*}$ satisfies (6) because by hypothesis $u_{1}^{*}$ satisfies (7). Indeed, substitution of the constructed $u^{*}$ into (6), where $\mathbf{B}=\mathbf{A}_{1} \mathbf{A}_{2}$, yields

$$
\mathbf{A}_{1} \mathbf{A}_{2} \mathbf{L} \mathbf{L}_{1}^{-1}\left(-f+\mathbf{A}_{1} \mathbf{L} u_{1}^{*}\right)=\mathbf{A}_{1} \mathbf{L} u_{1}^{*} .
$$

Taking into account operators commutativity, the following equality is valid

$$
\mathbf{A}_{1} \mathbf{L}\left\{\mathbf{A}_{2} \mathbf{L}_{1}^{-1}\left(-f+\mathbf{A}_{1} \mathbf{L} u_{1}^{*}\right)-u_{1}^{*}\right\}=0 .
$$

Since $\mathbf{A}_{1}$ and $\mathbf{L}$ are linear operators then it remains to verify that in (12) the element in brackets is zero. Since $u_{1}^{*} \in E_{1}$ satisfies (7), where $\mathbf{B}_{1}=\mathbf{A}_{2} \mathbf{A}_{1}$, then the following equality is valid

$$
\mathbf{A}_{2}\left(\mathbf{A}_{1} \mathbf{L} u_{1}^{*}-f\right)=\mathbf{L}_{1} u_{1}^{*}
$$

Hence $\mathbf{A}_{2}\left(\mathbf{A}_{1} \mathbf{L} u_{1}^{*}-f\right) \in \mathcal{R}\left(\mathbf{L}_{1}\right)$ and $u_{1}^{*}=\mathbf{L}_{1}^{-1} \mathbf{A}_{2}\left(\mathbf{A}_{1} \mathbf{L} u_{1}^{*}-f\right)$, where $\mathbf{L}_{1}^{-1}$ is the right inverse to the operator $\mathbf{L}_{1}$. Since $\mathbf{A}_{2} \mathbf{L}_{1}=\mathbf{L}_{1} \mathbf{A}_{2}$ then $\mathbf{A}_{2}=\mathbf{L}_{1}^{-1} \mathbf{A}_{2} \mathbf{L}_{1}$. This yields $\mathbf{A}_{2} \mathbf{L}_{1}^{-1}=\mathbf{L}_{1}^{-1} \mathbf{A}_{2} \mathbf{L}_{1} \mathbf{L}_{1}^{-1}$. From equality $\mathbf{L}_{1} \mathbf{L}_{1}^{-1}=\mathbf{I}$ it follows that the right inverse $\mathbf{L}_{1}^{-1}$ is also commutative with the operator $\mathbf{A}_{2}$. Then (13) can be represented as

$$
\mathbf{A}_{2} \mathbf{L}_{1}^{-1}\left(\mathbf{A}_{1} \mathbf{L} u_{1}^{*}-f\right)-u_{1}^{*}=0 .
$$

Thus we have shown that expression in brackets in (12) is zero. Lemma 3 is proved.

Let us concentrate on general case of skeleton chain of arbitrary finite length $p$. We assume operator $\mathbf{B}$ to have a skeleton chain $\left\{\mathbf{B}_{1}, \ldots, \mathbf{B}_{p}\right\}, p \geq 1$, and linear operators $\left\{A_{i}\right\}_{i=1}^{2 p}$ represent decomposition of $\mathbf{B}_{i}$ which skeleton is attached to $\mathbf{B}$. Introduce

$$
u_{i}=\prod_{j=1}^{i} \mathbf{A}_{2 j} u, i=1, \ldots, p
$$

where $u_{i} \in E_{i}, u_{i}=\mathbf{A}_{2 i} u_{i-1}, u_{0}:=u$.

If $u_{0}$ satisfies (6) then for $p \in \mathbb{N}$ by Definition 1 we get equalities

$$
\begin{gathered}
\mathbf{B}_{p} \mathbf{L} u_{p}=\mathbf{L}_{1} u_{p}+\prod_{j=1}^{p} \mathbf{A}_{2 j} f \\
\mathbf{L}_{1} u_{i}=-\prod_{j=1}^{i} \mathbf{A}_{2 j} f+\mathbf{A}_{2 i+1} \mathbf{L} u_{i+1}, \\
\mathbf{L}_{1} u=-f+\mathbf{A}_{1} \mathbf{L} u_{1} .
\end{gathered}
$$

For $p \geq 2$ there is a connection between the solution of (6) and system (15) - (17). In particular the following two lemmas can be formulated.

Lemma 4. Let $u^{*}$ satisfy (6) and operator $\mathbf{L}_{1}$ have left inverse. Then elements $u_{i}^{*}=$ $\prod_{j=1}^{i} \mathbf{A}_{2 j} u^{*}, i=p, p-1, \ldots, 1$ satisfy (15), (16), and $u^{*}$ satisfies (17).

Lemma 5. Let elements $u_{p}^{*}, u_{p-1}^{*}, \ldots, u_{1}^{*}, u^{*}$ satisfy (15) - (17) and operator $\mathbf{L}_{1}$ have right inverse. Then element $u^{*}$ determined from (17) of spitted system (15) - (17) is a solution to $(6)$. 
Proof of Lemma 4 and Lemma 5 for any natural number $p$ can be reduced to employment of the skeleton chain via operators $\left\{\mathbf{A}_{j}\right\}_{j=1}^{2 p}$ and repeats of stages of the proofs of Lemmas 2 and 3 for the case of $p=1$. Based on Lemmas 1-5 the following main result can be formulated.

Main Theorem. Let irreversible bounded operator $\mathbf{B}$ have a skeleton chain $\left\{B_{1}, \ldots, B_{p}\right\}$. Let operator $\mathbf{B}_{p} \mathbf{L}-\mathbf{L}_{1}$ have a bounded inverse with a domain in $E_{p}$. Let operator $\mathbf{L}_{1}$ be defined on domains $E_{i}, i=1, \ldots, p$ and $E$. Let operator $\mathbf{L}_{1}$ have an inverse bounded operator on $E_{i}, i=1, \ldots, p$ or $E$. Then system $(15)-(17)$ enjoys a unique solution $\left\{u_{p}^{*}, u_{p-1}^{*}, \ldots, u_{1}^{*}, u^{*}\right\}$, where

$$
\begin{gathered}
u_{p}^{*}=\left(\mathbf{B}_{p} \mathbf{L}-\mathbf{L}_{1}\right)^{-1} \prod_{j=1}^{p} \mathbf{A}_{2 j} f, \\
u_{i}^{*}=\mathbf{L}_{1}^{-1}\left\{-\prod_{j=1}^{i} \mathbf{A}_{2 j} f+\mathbf{A}_{2 j+1} \mathbf{L} u_{i+1}^{*}\right\}, i=p-1, \ldots, 1, \\
u^{*}=\mathbf{L}_{1}^{-1}\left\{-f+\mathbf{A}_{1} \mathbf{L} u_{1}^{*}\right\} .
\end{gathered}
$$

Moreover, element $u^{*}$ satisfies (6) and $u_{i}^{*}=\prod_{j=1}^{i} \mathbf{A}_{2 j} u^{*}, i=1, \ldots, p$.

By setting the initial-boundary conditions to ensure the reversibility of the operators $\mathbf{L}_{1}$ and $\mathbf{B}_{p} \mathbf{L}-\mathbf{L}_{1}$ with specific differential operators $\mathbf{L}$ and $\mathbf{L}_{1}$ and using the Main Theorem the existence and uniqueness theorems can be derived. Moreover, the formula obtained in Theorem can effectively build the desired classical solution of (1) with sufficient smoothness of $f: \Omega \subset \mathbb{R}^{m+1} \rightarrow E$ and the coefficients of the differential operators $\mathbf{L}$ and $\mathbf{L}_{1}$. Such applications of the theory are discussed further in section 3 .

\section{The Existence and Methods of Constructing Solutions of Nonclassic BVP with Partial Derivatives}

Consider the system

$$
\mathbf{B} \sum_{k_{1}+k_{2} \leq n} a_{k_{1} k_{2}} \frac{\partial^{k_{1}+k_{2}} u(x, t)}{\partial t^{k_{1}} \partial x^{k_{2}}}=\sum_{k_{1}+k_{2} \leq m} c_{k_{1} k_{2}} \frac{\partial^{k_{1}+k_{2}} u(x, t)}{\partial t^{k_{1}} \partial x^{k_{2}}}+f(x, t) .
$$

Here $m<n, \mathbf{B}$ is a constant $N \times N$ matrix, $\operatorname{det} \mathbf{B}=0, a_{k_{1} k_{2}}, c_{k_{1} k_{2}}$ are numbers, $a_{n 0 \neq 0,}, a_{0 n}=$ $0, c_{0 m} \neq 0, c_{m 0}=0$. The vector-functions $u(x, t)=\left(u_{1}(x, t), \ldots, u_{N}(x, t)\right)^{T}, f(x, t)=$ $\left(f_{1}(x, t), \ldots, f_{N}(x, t)\right)^{T}$ are supposed to be defined and analytical for $-\infty<x, t<\infty$.

Let rank $\mathbf{B}=r<N$. Then based on [11] $\mathbf{B}=\mathbf{A}_{1} \mathbf{A}_{2}$, where $\mathbf{A}_{1}$ is an $N \times r$ matrix, $\mathbf{A}_{2}$ is an $r \times N$ matrix. Let us introduce an $r \times r$ matrix $\mathbf{B}_{1}=\mathbf{A}_{2} \mathbf{A}_{1}$ and assume that $\operatorname{det} \mathbf{B}_{1} \neq 0$. Then taking into account Lemma 3 , the solution to (18) can be reduced to the successive solution of $(7),(8)$, which are in this case as follows:

$$
\begin{gathered}
\mathbf{B}_{1} \sum_{k_{1}+k_{2} \leq n} a_{k_{1} k_{2}} \frac{\partial^{k_{1}+k_{2}} u_{1}(x, t)}{\partial t^{k_{1}} \partial x^{k_{2}}}=\sum_{k_{1}+k_{2} \leq m} c_{k_{1} k_{2}} \frac{\partial^{k_{1}+k_{2}} u_{1}(x, t)}{\partial t^{k_{1}} \partial x^{k_{2}}}+\mathbf{A}_{2} f(x, t), \\
\sum_{k_{1}+k_{2} \leq m} c_{k_{1} k_{2}} \frac{\partial^{k_{1}+k_{2}} u(x, t)}{\partial t^{k_{1}} \partial x^{k_{2}}}=-f(x, t)+\mathbf{A}_{1} \sum_{k_{1}+k_{2} \leq n} a_{k_{1} k_{2}} \frac{\partial^{k_{1}+k_{2}} u_{1}(x, t)}{\partial t^{k_{1}} \partial x^{k_{2}}}
\end{gathered}
$$


where $\operatorname{det} \mathbf{B}_{1} \neq 0, u_{1}(x, t)=\left(u_{11}(x, t), \ldots, u_{1 r}(x, t)\right)^{T}, r<N, u_{1}=A_{2} u$. By hypothesis $a_{n 0} \neq 0, c_{0 m} \neq 0$, therefore for system (18) one may introduce the initial conditions

$$
\begin{gathered}
\left.\frac{\partial^{i} u(x, t)}{\partial x^{i}}\right|_{x=0}=0, i=0,1, \ldots, m-1, \\
\left.\mathbf{A}_{2} \frac{\partial^{i} u(x, t)}{\partial t^{i}}\right|_{t=0}=0, i=0,1, \ldots, n-1 .
\end{gathered}
$$

The vector-function $u_{1}(x, t)$ based on Kovalevskaya theorem can be defined as a unique solution to system (19) with initial conditions

$$
\left.\frac{\partial^{i} u_{1}(x, t)}{\partial t^{i}}\right|_{t=0}=0, i=0,1, \ldots, n-1 .
$$

By substitution of vector $u_{1}(x, t)$ into the right hand side of $(20)$, the desired vector $u(x, t)$ can be found as a unique solution to the Cauchy problem (20), (21).

Consider system

$$
\mathbf{B} \frac{\partial^{n} u(x, t)}{\partial x^{n}}=\left(\frac{\partial}{\partial t}-a^{2} \frac{\partial^{2}}{\partial x^{2}}\right) u(x, t)+f(x, t), n \geq 3 .
$$

As in system (18), $\mathbf{B}$ is a singular $N \times N$ matrix with $\operatorname{rank} \mathbf{B}=r<N, \mathbf{B}=\mathbf{A}_{1} \mathbf{A}_{2}, \mathbf{B}_{1}=$ $\mathbf{A}_{2} \mathbf{A}_{1}$, det $B_{1} \neq 0$. Let $f(x, t)=\left(f_{1}(x, t), \ldots, f_{N}(x, t)\right)^{T}$ be a vector-function defined for $0 \leq x \leq 1,0<t<\infty$, continuous with respect to $x$ and analytical by $t, u=\left(u_{1}, \ldots, u_{N}\right)^{T}$.

The objective is to construct a solution of (23) in $\Omega=\{0 \leq x \leq 1,0<t<\infty\}$. Based on Lemma 4 and the Main Theorem introduce system of two equations $\left(u_{1}=\mathbf{A}_{2} u\right)$

$$
\begin{gathered}
\mathbf{B}_{1} \frac{\partial^{n} u_{1}(x, t)}{\partial x^{n}}=\left(\frac{\partial}{\partial t}-a^{2} \frac{\partial^{2}}{\partial x^{2}}\right) u_{1}(x, t)+\mathbf{A}_{2} f(x, t), \\
\left(\frac{\partial}{\partial t}-a^{2} \frac{\partial^{2}}{\partial x^{2}}\right) u(x, t)=-f(x, t)+\mathbf{A}_{1} \frac{\partial^{n} u_{1}(x, t)}{\partial x^{n}}
\end{gathered}
$$

with initial-boundary conditions

$$
\begin{gathered}
\left.\frac{\partial^{i} u_{1}(x, t)}{\partial x^{i}}\right|_{x=0}=0, i=0,1, \ldots, n-1, \\
\left.u(x, t)\right|_{t=0}=0, \\
\left.u(x, t)\right|_{x=0}=0,\left.u(x, t)\right|_{x=1}=0 .
\end{gathered}
$$

Since det $B_{1} \neq 0$ then the vector-function $u_{1}(x, t)$ based on Kovalevskaya theorem can be defined as a unique solution of Cauchy problem (24) $-(26)$. Substitute $u_{1}(x, t)$ into the right hand side of (25). A unique solution of the first boundary value problem (25), (27), (28) is constructed for the heat equation using known formula (here readers may refer to p. 215 in [12]), using the source function. The constructed solution $u(x, t)$ will be a classic unique solution of (23) in domain $\Omega=\{0 \leq x \leq 1,0<t<\infty\}$. This solution satisfies the initial conditions

$$
\left.\mathbf{A}_{2} \frac{\partial^{i} u(x, t)}{\partial x^{i}}\right|_{x=0}=0, i=1, \ldots, n-1
$$

and (27), (28). 


\section{Skeleton Decomposition in the Theory of Irregular ODE in a Banach Space}

Consider the simplest irregular ODE

$$
\mathbf{B} \frac{d u(t)}{d t}=u(t)+f(t)
$$

$f(t):[0, \infty) \rightarrow E, \mathbf{B} \in \mathcal{L}(E \rightarrow E)$. Let $\left\{\mathbf{B}_{1}, \ldots, \mathbf{B}_{p}\right\}$ be a skeleton chain of the operator $\mathbf{B}$. Then from the Main Theorem the following results can be formulated.

Theorem 1. Let $\left\{\mathbf{B}_{1}, \ldots, \mathbf{B}_{p}\right\}$ be a regular skeleton chain, the function $f(t)$ be $(p-1)$ times differentiable. Then equation (29) with initial condition

$$
\left.\prod_{j=1}^{p} A_{2 j} u(t)\right|_{t=0}=c_{0}, c_{0} \in E_{p}
$$

enjoys a unique classic solution $u_{0}\left(t, c_{0}\right)$. Here

$$
u_{0}\left(t, c_{0}\right)=-f(t)+\mathbf{A}_{1} \frac{d u_{1}}{d t}
$$

where $u_{1}$ is defined uniquely (see the Main Theorem).

Let us outline the scheme for construction of the function $u_{1}\left(t, c_{0}\right)$ in solution (31) to problem (29), (30):

1. If $p=1$ then $u_{1}\left(t, c_{0}\right)$ satisfies the regular Cauchy problem

$$
\left\{\begin{array}{l}
\mathbf{B}_{1} \frac{d u_{1}}{d t}=u_{1}+\mathbf{A}_{2} f(t) \\
u_{1}(0)=c_{0}
\end{array}\right.
$$

2. If $p \geq 2$ then the function $u_{1}\left(t, c_{0}\right)$ can be constructed by the following recursion

$$
\begin{gathered}
\left\{\begin{array}{l}
\mathbf{B}_{p} \frac{d u_{p}}{d t}=u_{p}+\prod_{j=1}^{p} \mathbf{A}_{2 j} f(t), \\
u_{p}(0)=c_{0} .
\end{array}\right. \\
u_{i}\left(t, c_{0}\right)=\mathbf{A}_{2 i+1} \frac{d u_{i+1}\left(t, c_{0}\right)}{d t}-\prod_{j=1}^{i} \mathbf{A}_{2 j} f(t), i=p-1, p-2, \ldots, 1 .
\end{gathered}
$$

Theorem 2. Let $\left\{\mathbf{B}_{1}, \ldots, \mathbf{B}_{p-1}, 0\right\}$ be a singular chain of length $p \geq 1,0$ is a null operator acting from $E_{p}$ to $E_{p}$. Then $\mathbf{B}$ is a nilpotent operator and the homogeneous equation $\mathbf{B} \frac{d u}{d t}=u$ has only trivial solution. In this case, if the function $f(t)$ is p-times differentiable then the unique classic solution of (29) can be constructed as follows

$$
u_{n}(t)=-f(t)+\mathbf{B} \frac{d}{d t} u_{n-1}(t), u_{0}(t)=-f(t), n=1,2, \ldots, p .
$$

Here the function $u_{p}(t)$ is a unique classic solution of (29). 


\section{Conclusion}

This paper reports on the novel method of skeleton chains initiated in [4] for the linear operators in order to produce new non-classical boundary value problems for systems of differential and integral-differential equations with partial derivatives arising in modern mathematical modelling.

Acknowledgements. This work is fuilfilled within International Science and Technology Cooperation Program (No. 2015DFA70850) of China \& Russia; NSFC Grant No. 61673398. The first author's work is partly supported by RSF Grant No. 14-19-00054.

\section{References}

1. Petrowsky I.G., Oleinik O.A. Selected Works. Part I: Systems of Partial Differential Equations and Algebraic Geometry. Amsterdam, Gordon and Breach Publishers, 1996.

2. Voropai N.I., Kurbatsky V.G., Tomin N.V., Panasetsky D.A., Sidorov D.N. Complex intellektualnih sredstv dlya predotvrashenia krupnih avarii $v$ elektroenergenicheskih sistemah [Intellectual Algorithms for Major Accidents Prevention in Electric Power Systems]. Novosibirsk, Nauka, 2016.

3. Sidorov N.A. Obshchie voprosy regulyarizatsii $v$ zadachakh teorii vetvleniya [General Regularization Questions in Problems of Bifurcation Theory]. Irkutsk, Izdatel'stvo Irkutskogo Universiteta, 1982.

4. Sidorov N., Sidorov D., Li Y. Skeleton Decomposition of Linear Operators in the Theory of Degenerate Differential Equations. 2015. arXiv:1511.08976. 4 p.

5. Sidorov N., Loginov B., Sinitsyn A., Falaleev M. Lyapunov - Schmidt Methods in Nonlinear Analysis and Applications. Springer Netherlands, 2013. DOI 10.1007/978-94-017-2122-6

6. Sviridyuk G.A., Fedorov V.E. Linear Sobolev Type Equations and Degenerate Semigroups of Operators. Utrecht, Boston, Köln, VSP, 2003. DOI: 10.1515/9783110915501

7. Zamyshlyaeva A.A., Tsyplenkova O.N. Optimal Control of Solutions of the Showalter Sidorov - Dirichlet Problem for the Boussinesq - Löve Equation. Differential Equations, 2013, vol. 49, issue 11, pp. 1356-1365. DOI: 10.1134/S0012266113110049

8. Keller A.V., Shestakov A.L., Sviridyuk G.A., Khudyakov Yu.V. The Numerical Algorithms for the Measurement of the Deterministic and Stochastic Signals. Semigroups of Operators Theory and Applications, Springer International Publishing, 2015, vol. 113, pp. 183-195.

9. Sidorov D. Integral Dynamical Models: Singularities, Signals and Control. Singapore, London, World Scientific, 2015.

10. Li Y., Han J., Cao Y., Li Yu., Xiong J., Sidorov D., Panasetsky D. A Modular Multilevel Converter Type Solid State Transformer with Internal Model Control Method. International Journal of Electrical Power \& Energy Systems, 2017, vol. 85, pp. 153-163. DOI: $10.1016 /$ j.ijepes.2016.09.001

11. Gantmacher F.R. The Theory of Matrices. N.-Y., Chelsea, 1959.

12. Tikhonov A.N., Samarskii A.A. Equations of Mathematical Physics. Courier Corporation, 2013.

13. Sobolev S.L. [The Cauchy Problem for a Special Case of System That Are not of Kovalevskaya Type]. Doklady akademii nauk SSSR, 1952, vol. 82, no. 2, pp. 1007-1009. (in Russian) 
14. Loginov B.V., Rousak Yu.B., Kim-Tyan L.R. Differential Equations with Degenerate, Depending on the Unknown Function Operator at the Derivative. Proceedings of the Seventh International Conference on Differential and Functional-Differential Equations, 2016, pp. 119-147.

Received 28 December, 2016

УДК 517.9

DOI: $10.14529 / \mathrm{mmp} 170205$

\section{О ПОСТРОЕНИИ РЕШЕНИЙ НЕРЕГУЛЯРНЫХ СИСТЕМ С ЧАСТНЫМИ ПРОИЗВОДНЫМИ НА ОСНОВЕ ТЕОРИИ СКЕЛЕТНЫХ РАЗЛОЖЕНИЙ ЛИНЕЙНЫХ ОПЕРАТОРОВ}

\section{Д.Н. Сидоров ${ }^{1,2}$, H.A. Сидоров ${ }^{3}$}

${ }^{1}$ Институт систем энергетики имени Л.А. Мелентьева СО РАН, г. Иркутск

${ }^{2}$ Иркутский национальный исследовательский технический университет, г. Иркутск

${ }^{3}$ Иркутский государственный университет, г. Иркутск

Рассматриваются линейные системы уравнений с частными производными. В главной части систем стоит линейный необратимый оператор, допускающий скелетное разложение. Входящие в систему дифференциальные операторы имеют достаточно гладкие коэффициенты. Области определения дифференциальных операторов в конкретных ситуациях, рассмотренных в работе, состоят из линейных многообразий достаточно гладких функций со значениями в банаховом пространстве подчиненных дополнительным граничным условиям. Вводится понятие скелетной цепочки линейного оператора, стоящего в главной части системы. Предполагается, что этот оператор порождает скелетную цепочку конечной длины. В этом случае решение исходной системы сводится к регулярной расщепленной системе уравнений, разрешенных относительно старших дифференциальных выражений с определенными начально-краевыми условиями. Указаны возможные обобщения предложенного подхода и рассмотрено его приложение к постановке граничных задач в нелинейном случае. Результаты дополняют элементы теории дифференциальных уравнений с вырождениями, заложенные в монографиях MR 87a:58036, Zbl 1027.47001.

Ключевые слова: некорректная задача; задача Коши; необратимый оператор; скелетное разложение; скелетные цепочки, граничные задачи.

Денис Николаевич Сидоров, доктор физико-математических наук, профессор, Институт систем энергетики имени Л.А. Мелентьева СО РАН, Иркутский национальный исследовательский технический университет (г. Иркутск, Российская Федерация), dsidorov@isem.irk.ru.

Николай Александрович Сидоров, доктор физико-математических наук, професcop, Иркутский государственный университет (г. Иркутск, Российская Федерация), sidorovisu@gmail.com.

Поступила в редакиию 28 декабря 2016 г.

Вестник ЮУрГУ. Серия «Математическое моделирование

и программирование» (Вестник ЮУрГУ ММП). 2017. Т. 10, № 2. С. 63-73 